\title{
Timed Up and Go Test With a Cognitive Task: Correlations With Neuropsychological Measures in People With Parkinson's Disease
}

Kübra Çekok ${ }^{1,} 2$, Turhan Kahraman ${ }^{3}$, Gözde Duran ${ }^{4}$, Berril Dönmez Çolakoğlu ${ }^{5}$, Görsev Yener 5, 6 , Deniz Yerlikaya ${ }^{7}$, Arzu Genç ${ }^{8}$

1. Physical Therapy, Medical Park Hospital, Izmir, TUR 2. Physical Therapy, Graduate School of Health Sciences, Dokuz Eylül University, Izmir, TUR 3. Physiotherapy and Rehabilitation, Izmir Katip Celebi University, Izmir, TUR 4. Physical Medicine and Rehabilitation, Graduate School of Health Sciences, Dokuz Eylül University, Izmir, TUR 5. Neurology, Dokuz Eylül University, Izmir, TUR 6. Izmir Biomedicine and Genome Center, Dokuz Eylül University, Izmir, TUR 7. Psychology, Dokuz Eylül University, Izmir, TUR 8. Physical Therapy and Rehabilitation, Dokuz Eylül University, Izmir, TUR

Corresponding author: Kübra Çekok, kbrackk@gmail.com

\section{Abstract}

\section{Background}

The Timed Up and Go (TUG) test is a simple and widely used clinical test for the assessment of lower extremity function, balance, mobility, and fall risk in various populations. The TUG has been found as a valid and reliable measure in people with Parkinson's disease (PD). Besides, the addition of a cognitive task to the TUG (TUG-cognitive) enhances predictive validity related to fall risk in people with PD. However, further investigation is needed about the correlations of the TUG-cognitive test with neuropsychological measures in people with PD.

\section{Methods}

Thirty-three people with PD [modified Hoehn and Yahr scale, median (min-max)=2.5 (1.0-3.0)] participated in this cross-sectional study. The TUG was administered in the traditional way and with a cognitive task (counting backward by three from any number between 20 and 100). Neuropsychological measures included the Montreal Cognitive Assessment (MoCA), Trail Making Test (TMT), and the Simple Reaction Time (SRT) test for stepping. The self-reported number of falls in the last six months was also recorded.

\section{Results}

The TUG-cognitive [13.1 ( $\mathrm{SD}=8.5$ ) seconds] was significantly longer than the TUG-traditional [12.2 ( $\mathrm{SD}=8.1)$ seconds] $(\mathrm{p}<0.01)$. The TUG-cognitive significantly correlated with the MoCA [(rho=-0.712), TMT part A (TMT-A; rho=0.722), TMT part B (TMT-B; rho=0.694), SRT (rho=0.794), and number of falls (rho=0.960)] $(\mathrm{p}<0.01)$. The TUG-traditional also significantly correlated with the MoCA (rho $=-0.682)$, TMT-A ( $r h o=0.684)$,

Received 09/03/2020

Review began 09/07/2020 Review ended 09/18/2020 Published 09/22/2020

\section{() Copyright 2020}

Çekok et al. This is an open access article distributed under the terms of the Creative Commons Attribution License CC-BY 4.0., which permits unrestricted use, distribution, and reproduction in any medium, provided the original author and source are credited. TMT-B (rho=0.746), SRT (rho=0.755), and number of falls (rho=0.702) $(\mathrm{p}<0.01)$.

\section{Conclusion}

Both the TUG-cognitive and TUG-traditional strongly correlated with neuropsychological measures; while the correlations were slightly stronger for the TUG-cognitive, the difference was not significant. The TUGcognitive can be used in the clinical practice as a simple and more informative alternative to the TUGtraditional in people with PD.

Categories: Neurology, Physical Medicine \& Rehabilitation

Keywords: parkinson's disease, neuropsychological tests, reaction time, executive function, postural balance

\section{Introduction}

Many activities in daily life require individuals to perform more than one task at the same time, which is called dual-tasking. Adequate balance, coordination, attention, and judgment are required while performing dual-tasks, which require the motor and cognitive systems to work together [1]. Most falls in older people are associated with decreased dual-task performance [2]. Dual-task performance is also adversely affected in people with Parkinson's disease (PD).

In people with $\mathrm{PD}$, many walking features such as decreased swing time, walking speed, and stride length tend to deteriorate. In addition, coordination and timing problems are also frequent in people with PD [3]. Clinical walking tests create a map for detecting fall risk and can help with health promotion strategies. The Timed Up and Go (TUG) test is one of the most frequently used clinical walking tests in people with PD. The TUG includes many activities that are common in daily life, such as sit-to-stand, walking, and turning. The 
To increase the TUG's precision, additional tasks such as motor and cognitive tasks can be added to the traditional TUG test. Lundin-Olsson et al. showed that the TUG with a cognitive task (TUG-cognitive) (counting back by three) was more sensitive and specific in predicting the rate of falls in older people [5]. Many people with PD have cognitive dysfunction, which affects walking and balance and thereby increasing fall risk [6]. Therefore, it is suggested that functional motor skills should be evaluated under a variety of dual-task conditions such as in the TUG-cognitive in people with PD [7]. However, research examining the correlation between the TUG-cognitive and neuropsychological measures in people with PD is scarce. Therefore, this study aimed to investigate the correlations of the TUG-cognitive test with the neuropsychological measures by comparing it with the TUG-traditional in people with PD. Due to the nature of the dual-task condition, we hypothesized that the TUG-cognitive would have greater correlations with neuropsychological measures compared to the TUG-traditional.

\section{Materials And Methods \\ Design, setting, and participants}

This cross-sectional study involved a one-time assessment of people with PD. The demographic information (age, gender, education duration, and body mass index) was gathered using face-to-face interviews. The self-reported number of falls in the last six months was also recorded. The modified Hoehn and Yahr scale and the Unified Parkinson's Disease Rating Scale (UPDRS) were applied to assess disability and symptom severity, respectively. The Hoehn and Yahr scale is used to evaluate disability and impairment related to clinical disease progression of PD. The scale originally included stages 1 through 5 [8]. The Hoehn and Yahr scale was modified with the addition of stages 1.5 and 2.5 to account for the intermediate course of PD. The stages range from 1 (unilateral involvement only) to 5 (wheelchair-bound or bedridden unless aided). The UPDRS includes series of ratings for typical symptoms of PD that are assessed in different parts: part 1 (mental dysfunction and mood), part 2 (activities of daily living), part 3 (motor section), and part 4 (treatment-related complications). Higher scores in the UPDRS indicate higher symptom severity [9].

We recruited 33 people with idiopathic PD from the Movement Disorders Clinic of Dokuz Eylül University, Izmir, Turkey. The inclusion criteria were as follows: people living in the community, ability to walk unaided for $10 \mathrm{~m}$, and having modified Hoehn and Yahr stages 1-3. People with any medical conditions that would preclude or interfere with the TUG assessments (e.g., physician-diagnosed dementia, acute or terminal illness, progressive neurodegenerative diseases other than $\mathrm{PD}$, major psychiatric illnesses, visual impairments that could not be corrected, and deep brain stimulation) were excluded from the study [10].

In a previous study, a strong correlation (rho $\approx 0.7$ ) was observed between the Montreal Cognitive Assessment (MoCA) and the TUG-cognitive in people with PD [11]. The required sample size was calculated as 11 for alpha error probability=0.05 and power=0.80 using $G^{*}$ Power (version 3.1.9.2, Dusseldorf University, Germany). Since the number was relatively small, we decided to increase the number by three times (i.e., 33 participants).

\section{Ethical approval}

The ethical approval for this study was obtained from the Noninvasive Research Ethics Board of Dokuz Eylül University (2018/26-10). The participants provided written consent prior to their participation in the study.

\section{Outcome measures}

Neuropsychological Evaluation

The MoCA was used to assess global cognition. The MoCA is a screening tool for mild cognitive impairment and assesses a variety of cognitive domains including attention, memory, executive function, visuospatial ability, and language. The MoCA had adequate psychometric properties in people with PD [12]. The scores on the MoCA ranged from 0 to 30 and higher scores indicated better cognitive function. The Turkish version of the MoCA also showed acceptable psychometric properties in people with PD [13].

Executive function was assessed by the Trail Making Test (TMT), which is a well-established neuropsychological screening tool with high validity and reliability [14]. The TMT has two parts. Part A (TMT-A) is a visual-scanning task and part B (TMT-B) adds to it a measure of cognitive flexibility. In the TMT-A, the participants were asked to draw lines sequentially connecting consecutively numbered circles from 1 to 25 , which were randomly arranged on a page as quickly as possible. In the TMT-B, the participants were asked to connect the same number of circles in an alternating sequence of numbers and letters. The standardized Turkish version of the TMT was also available and it showed good psychometric properties [14].

The Multi-Operational Apparatus for Reaction Time (MOART; Lafayette Instrument Company, Lafayette, IN) was used with food-pads to carry out the Simple Reaction Time (SRT) test for stepping [15]. A number of practice trials were administered for familiarization with the procedure [15]. Participants performed five 
stepping trials and the reaction time (time taken from receipt of the stimulus to lifting the stepping foot off of the foot-pad) was recorded for each trial as milliseconds [15]. There were 10-second rest intervals between the trials [15]. The SRS score was computed as the sum of five trials [15].

TUG Assessments

The TUG-traditional and TUG-cognitive were administered in random order for each patient. Each test was repeated three times and the average of the duration was used for data analysis. The TUG-traditional test was applied by making the participants perform the following tasks: standing from a chair, walking $3 \mathrm{~m}$, turning around, returning to the chair, and sitting down. Although there are many different dual-task conditions for the TUG, such as delayed memory task, digit span forward, and serial subtraction [11,16], digit span-backward is one of the most commonly used version in clinical practice [17]. Therefore, in the current study, the TUG-cognitive was applied while counting backward by three from any number between 20 and $100[17]$.

\section{Statistical analysis}

Data were analyzed using IBM SPSS Statistics for Windows version 24.0 (IBM Corp., Armonk, NY). Descriptive statistics were used to describe participants' demographic characteristics and assessment results. Checking of the normal distribution of the data was applied using the Shapiro-Wilk test and investigation of histogram graphics. Correlations between the study variables were assessed using Spearman's rank-order correlation analysis. The correlation coefficients were interpreted as strong $(>0.5)$, moderate (0.3-0.5), and weak (0.2-0.3) [18]. The significance of the difference between the two correlation coefficients was calculated using the Fisher r-to-z transformation. Statistical significance was defined at a pvalue of $<0.05$.

\section{Results}

There were 33 participants; the mean age of the participants was $67.9(\mathrm{SD}=7.9)$ years, and $73 \%$ of them were male. The UPDRS score was $35.4(\mathrm{SD}=15.6)$ and the median of the modified Hoehn and Yahr stage was 2.5 (min-max=1.0-3.0). Seventeen participants (51.5\%) had a fall history within the last six months and the median number of falls was 1 . Table 1 presents the demographic characteristics and outcome measure results of the participants. 


\section{Cureus}

\section{Variables}

Gender, n (\%)

Female

Male

Body mass index, kg/m², mean (SD)

Education duration, years, mean (SD)

Number of falls in the last 6 months, median (min-max)

UPDRS, mean (SD)

Modified Hoehn and Yahr scale, median (min-max)

TMT-A, seconds, mean (SD)

TMT-B, seconds, mean (SD)

$124.9(58.4)$

SRT, milliseconds, mean (SD)

The TUG-cognitive [13.1 ( $\mathrm{SD}=8.5$ ) seconds] was significantly longer than the TUG-traditional [12.2 $(\mathrm{SD}=8.1)$ seconds] $(\mathrm{p}<0.01)$. The TUG-cognitive and TUG-traditional strongly correlated with each other $(\mathrm{rho}=0.941$, $\mathrm{p}<0.01)$. The TUG-cognitive significantly correlated with the MoCA ( $r$ ho $=-0.712)$, TMT-A (rho $=0.722)$, TMT-B (rho=0.694), SRT (rho=0.794), and number of falls in the last six months $(r h o=0.696)(p<0.01)$. The TUGtraditional also significantly correlated with the MoCA (rho=-0.682), TMT-A (rho=0.684), TMT-B (rho=0.746), $\mathrm{SRT}(\mathrm{rho}=0.755)$, and number of falls in the last six months $(\mathrm{rho}=0.702)(\mathrm{p}<0.01)$. None of the correlation coefficients was significantly different between the TUG-traditional and TUG-cognitive ( $p>0.05$ ). Table 2 presents the correlations between TUG tests and neuropsychological measures. 


\begin{tabular}{|c|c|c|c|c|c|}
\hline \multirow[b]{2}{*}{ Variable } & \multicolumn{2}{|c|}{ TUG-cognitive } & \multicolumn{2}{|c|}{ TUG-traditional } & \multirow[b]{2}{*}{ P-value ${ }^{a}$} \\
\hline & rho & P-value & rho & P-value & \\
\hline TUG-cognitive & - & - & 0.941 & $<0.001^{\star}$ & - \\
\hline SRT & 0.794 & $<0.001^{*}$ & 0.755 & $<0.001^{*}$ & 0.703 \\
\hline TMT-A & 0.722 & $<0.001^{*}$ & 0.684 & $<0.001^{*}$ & 0.771 \\
\hline TMT-B & 0.694 & $<0.001^{\star}$ & 0.746 & $<0.001^{*}$ & 0.675 \\
\hline MoCA & -0.712 & $<0.001^{\star}$ & -0.682 & $<0.001^{*}$ & 0.818 \\
\hline Number of falls in the last 6 months & 0.696 & $<0.001^{\star}$ & 0.702 & $<0.001^{*}$ & 0.960 \\
\hline
\end{tabular}

TABLE 2: Correlations between TUG tests and neuropsychological measures in people with PD $(\mathrm{N}=33)$

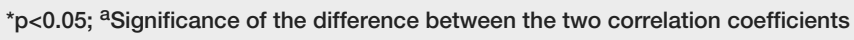

PD: Parkinson's disease; TUG: Timed Up and Go test; MoCA: Montreal Cognitive Assessment; TMT: Trail Making Test; SRT: Simple Reaction Time; (): not reported

\section{Discussion}

In the current study, we have shown that both the TUG-traditional and TUG-cognitive correlated with different neuropsychological measures and the number of falls in the people with PD. Moreover, the TUGcognitive had a slightly greater correlation coefficient with the cognitive function, visual attention and task switching, and reaction time compared to the TUG-traditional. However, there was no significant difference between the correlation coefficients.

The completion time of the TUG-cognitive was significantly longer than that of the TUG-traditional in people with $\mathrm{PD}$. In the walking tests, cognitive and motor regions of the brain work together. As the second task is assigned in the TUG-cognitive test, the process becomes complicated and the walking speed is affected. This result can be interpreted according to the capacity theory [19]. When two tasks are performed at the same time, competition within the limited resources causes deterioration in the performance of the single or both tasks. Walking assessment with a cognitive task requiring cognitive flexibility is important since cognitive flexibility is an early cognitive impairment in people with PD [20]. The current study has demonstrated that the TUG-cognitive test had more completion time compared to the TUG-traditional test. Therefore, the TUG-cognitive could be chosen as a simple clinical test that can show impairment in the dualtask performance and cognitive function in the people with PD.

Our study also showed that TUG-cognitive significantly correlated with the cognitive function as assessed by the MoCA, which is an internationally accepted tool that is frequently used in clinical and research settings [21]. However, it has been emphasized in some studies that the limitation of the MoCA in evaluating executive function and attention should be taken into consideration [22]. Therefore, we also investigated the correlations of visual attention and task switching, as well as reaction time for stepping. We demonstrated that TUG-cognitive significantly correlated with cognitive function, visual attention and task switching, and reaction time. In people with $\mathrm{PD}$, early attention and executive dysfunctions result from basal ganglion pathology, and they have difficulty in appropriately performing a dual-task of cognitive performance. Therefore, it is inevitable that dual-task performance and executive function and attention should be related [23]. They are effective components of executive functions on will, self-awareness, planning, focus, monitoring response, and walking attention [24]. Impairment of one or more of the executive function components affects a person's ability to walk safely and effectively [25]. Therefore, impaired executive function leads to difficulties in dual-task conditions and, consequently, increases fall risk.

It has been shown that prolonged reaction time is an independent risk factor for falls in people with PD [26]. Intact cognitive function is important for fall avoidance by providing safe foot placement and may elucidate why reduced cognitive performance has been strongly associated with falls in people with PD [27]. Therefore, simple clinical assessment methods that significantly correlate with the above-mentioned parameters are important to quickly evaluate people with PD and to consult them for further detailed assessments. Both the TUG-traditional and TUG-cognitive significantly correlated with several important neuropsychological aspects including reaction time for stepping and number of falls within the last six months. Although there was no significant difference between the correlation coefficients, the TUGcognitive had slightly greater correlation coefficients compared to the TUG-traditional. In addition, the 
completion time of the TUG-cognitive was significantly longer than that of the TUG-traditional. We speculate that TUG-cognitive would have significantly greater correlations compared to the TUG-traditional in people with PD having more disease severity, which should be investigated in future studies. In addition, due to the mild disability levels of the participants, counting backward by three as a cognitive task could be easy for the participants. A more complex attention activity such as a serial subtraction test would be better in such populations [16]. In future studies, different protocols for cognitive demand in the TUG should be investigated. Nevertheless, the TUG-cognitive can be used in clinical practice as a simple and more informative alternative to the TUG-traditional in people with PD.

Dual-task performance is affected by many factors such as balance, cognitive performance, cognitive load, walking ability, and environmental factors [28]. Therefore, psychometrically sound assessment methods are highly required. In this study, we have shown significant correlations of TUG-cognitive with several neuropsychological assessments and the number of falls, which supports its validity in people with PD.

\section{Limitations}

This study has some limitations. Firstly, there was the absence of a control group, and having a healthy control group would be better to demonstrate whether the observed correlations were specific to people with PD. Secondly, the participants had mild to moderate disease severity, which limits the generalizability of our results. Finally, the current study supports that the TUG-cognitive has robust construct validity against neuropsychological measures in people with PD. However, the different important psychometric properties of the TUG-cognitive such as test-retest and interrater reliability, discriminant validity, and minimal clinically important difference should be investigated in future studies.

\section{Conclusions}

Both the TUG-cognitive and TUG-traditional strongly correlated with the neuropsychological measures and the number of falls, yet the TUG-cognitive had slightly greater associations with some of the neuropsychological measures. People with PD showed significantly less performance levels in the TUGcognitive compared to the TUG-traditional. The TUG-cognitive can be used in clinical practice as a simple and more informative alternative to the TUG-traditional in people with PD. However, we speculate that the TUG-cognitive would have significantly greater correlations compared to the TUG-traditional in PD patients with higher disease severity, which should be investigated in future studies.

\section{Additional Information \\ Disclosures}

Human subjects: Consent was obtained by all participants in this study. Noninvasive Research Ethics Board of Dokuz Eylül University issued approval 2018/26-10. This study received ethical approval from the Noninvasive Research Ethics Board of Dokuz Eylül University (2018/26-10). . Animal subjects: All authors have confirmed that this study did not involve animal subjects or tissue. Conflicts of interest: In compliance with the ICMJE uniform disclosure form, all authors declare the following: Payment/services info: This research was funded by Department of Scientific Research Projects, Dokuz Eylül University (Project ID: 2019.KB.SAG.033). Financial relationships: All authors have declared that they have no financial relationships at present or within the previous three years with any organizations that might have an interest in the submitted work. Other relationships: All authors have declared that there are no other relationships or activities that could appear to have influenced the submitted work.

\section{References}

1. Floriano EN, Alves JF, de Almeida IA, de Souza RB, Christofoletti G, Santos SM: Dual task performance: a comparison between healthy elderly individuals and those with Parkinson's disease. Fisioter Mov. 2015, 28:251-258. 10.1590/0103-5150.028.002.A005

2. Kelly VE, Eusterbrock AJ, Shumway-Cook A: A review of dual-task walking deficits in people with Parkinson's disease: motor and cognitive contributions, mechanisms, and clinical implications. Parkinsons Dis. 2012, 2012:918719. 10.1155/2012/918719

3. Hausdorff JM: Gait dynamics in Parkinson's disease: common and distinct behavior among stride length, gait variability, and fractal-like scaling. Chaos. 2009, 19:026113. 10.1063/1.3147408

4. Morris S, Morris ME, Iansek R: Reliability of measurements obtained with the Timed "Up \& Go" test in people with Parkinson disease. Phys Ther. 2001, 81:810-818. 10.1093/ptj/81.2.810

5. Lundin-Olsson L, Nyberg L, Gustafson Y: Attention, frailty, and falls: the effect of a manual task on basic mobility. J Am Geriatr Soc. 1998, 46:758-761. 10.1111/j.1532-5415.1998.tb03813.x

6. Chen TY, Peronto CL, Edwards JD: Cognitive function as a prospective predictor of falls . J Gerontol B Psychol Sci Soc Sci. 2012, 67:720-728. 10.1093/geronb/gbs052

7. van Iersel MB, Kessels RP, Bloem BR, Verbeek AL, Olde Rikkert MG: Executive functions are associated with gait and balance in community-living elderly people. J Gerontol A Biol Sci Med Sci. 2008, 63:1344-1349. 10.1093/gerona/63.12.1344

8. Hoehn MM, Yahr MD: Parkinsonism: onset, progression and mortality. Neurology. 1967, 17:427-442. 10.1212/wnl.17.5.427

9. Logemann JA, Fisher HB, Boshes B, Blonsky ER: Frequency and cooccurrence of vocal tract dysfunctions in 
the speech of a large sample of Parkinson patients. J Speech Hear Disord. 1978, 43:47-57. 10.1044/jshd.4301.47

10. Caetano MJD, Lord SR, Allen NE, Song J, Paul SS, Canning CG, Menant JCC: Executive functioning, muscle power and reactive balance are major contributors to gait adaptability in people with Parkinson's disease. Front Aging Neurosci. 2019, 11:154. 10.3389/fnagi.2019.00154

11. Varalta V, Picelli A, Fonte C, et al.: Relationship between cognitive performance and motor dysfunction in patients with Parkinson's disease: a pilot cross-sectional study. Biomed Res Int. 2015, 2015:365959. $10.1155 / 2015 / 365959$

12. Hoops S, Nazem S, Siderowf AD, Duda JE, Xie SX, Stern MB, Weintraub D: Validity of the MoCA and MMSE in the detection of MCI and dementia in Parkinson disease. Neurology. 2009, 73:1738-1745. 10.1212/WNL.0b013e3181c34b47

13. Ozdilek B, Kenangil G: Validation of the Turkish Version of the Montreal Cognitive Assessment Scale (MoCA-TR) in patients with Parkinson's disease. Clin Neuropsychol. 2014, 28:333-343. 10.1080/13854046.2014.881554

14. Cangoz B, Karakoc E, Selekler K: Trail Making Test: normative data for Turkish elderly population by age, sex and education. J Neurol Sci. 2009, 283:73-78. 10.1016/j.jns.2009.02.313

15. Goldberg A, Schepens SL, Feely SM, Garbern JY, Miller LJ, Siskind CE, Conti GE: Deficits in stepping response time are associated with impairments in balance and mobility in people with Huntington disease. J Neurol Sci. 2010, 298:91-95. 10.1016/j.jns.2010.08.002

16. Zirek E, Ersoz Huseyinsinoglu B, Tufekcioglu Z, Bilgic B, Hanagasi H: Which cognitive dual-task walking causes most interference on the Timed Up and Go test in Parkinson's disease: a controlled study. Neurol Sci. 2018, 39:2151-2157. 10.1007/s10072-018-3564-2

17. Maranhão-Filho PA, Maranhão ET, Lima MA, Silva MM: Rethinking the neurological examination II: dynamic balance assessment. Arq Neuropsiquiatr. 2011, 69:959-963. 10.1590/s0004-282x2011000700022

18. Cohen J: Statistical Power Analysis for the Behavioral Sciences (2nd ed.). L Erlbaum Associates, Hillsdale, NJ; 1998.

19. Tombu M, Jolicoeur P: A central capacity sharing model of dual-task performance. J Exp Psychol Hum Percept Perform. 2003, 29:3-18. 10.1037//0096-1523.29.1.3

20. Belghali M, Chastan N, Davenne D, Decker LM: Improving dual-task walking paradigms to detect prodromal Parkinson's and Alzheimer's diseases. Front Neurol. 2017, 8:207. 10.3389/fneur.2017.00207

21. Hu MT, Szewczyk-Królikowski K, Tomlinson P, et al.: Predictors of cognitive impairment in an early stage Parkinson's disease cohort. Mov Disord. 2014, 29:351-359. 10.1002/mds.25748

22. Yogev-Seligmann G, Hausdorff JM, Giladi N: The role of executive function and attention in gait . Mov Disord. 2008, 23:329-342. 10.1002/mds.21720

23. Hausdorff JM, Doniger GM, Springer S, Yogev G, Simon ES, Giladi N: A common cognitive profile in elderly fallers and in patients with Parkinson's disease: the prominence of impaired executive function and attention. Exp Aging Res. 2006, 32:411-429. 10.1080/03610730600875817

24. Hausdorff JM, Schweiger A, Herman T, Yogev-Seligmann G, Giladi N: Dual-task decrements in gait: contributing factors among healthy older adults. J Gerontol A Biol Sci Med Sci. 2008, 63:1335-1343. 10.1093/gerona/63.12.1335

25. Plotnik M, Dagan Y, Gurevich T, Giladi N, Hausdorff JM: Effects of cognitive function on gait and dual tasking abilities in patients with Parkinson's disease suffering from motor response fluctuations. Exp Brain Res. 2011, 208:169-179. 10.1007/s00221-010-2469-y

26. Jahanshahi M, Brown RG, Marsden CD: The effect of withdrawal of dopaminergic medication on simple and choice reaction time and the use of advance information in Parkinson's disease. J Neurol Neurosurg Psychiatry. 1992, 55:1168-1176. 10.1136/jnnp.55.12.1168

27. Paul SS, Sherrington C, Canning CG, Fung VS, Close JC, Lord SR: The relative contribution of physical and cognitive fall risk factors in people with Parkinson's disease: a large prospective cohort study. Neurorehabil Neural Repair. 2014, 28:282-290. 10.1177/1545968313508470

28. Rochester L, Galna B, Lord S, Burn D: The nature of dual-task interference during gait in incident Parkinson's disease. Neuroscience. 2014, 265:83-94. 10.1016/j.neuroscience.2014.01.041 